\title{
COMMUTATIVE SUBDIRECTLY IRREDUCIBLE RINGS
}

\author{
NATHAN DIVINSKY
}

Introduction. A subdirectly irreducible ring is one in which the intersection of all the nonzero ideals is a nonzero ideal. Such rings are important not only because every ring is isomorphic to a subdirect sum of subdirectly irreducible rings, but also because the theory of rings without chain conditions uses the concept heavily. Our major knowledge of such rings is contained in $[1],{ }^{1}$ where Professor McCoy showed that every commutative subdirectly irreducible ring is one of three kinds. We shall classify them as

Type $(\alpha)$. Fields.

Type $(\beta)$. Every element is a divisor of zero.

Type $(\gamma)$. There exist both nondivisors of zero and nilpotent elements.

We shall restrict ourselves to the commutative case and henceforth not repeat its assumption. We shall employ the following notation:

$A=$ the commutative subdirectly irreducible ring.

$D=$ the set of all divisors of zero of $A$.

$J=$ the Jacobson radical of $A$.

$N=$ the maximal nilideal of $A$.

$N^{*}=$ the set of elements that annihilate $N$.

$D^{*}=$ the set of elements that annihilate $D$.

$Q=$ the unique minimal ideal of $A$ that is contained in every nonzero ideal of $A$.

As in [2] we shall say that $A$ is bound to its maximal nilideal if $N^{*} \leqq N$.

Rings of type $(\beta)$ were considered in [2] and it was shown that they are bound to $N$. In addition if they satisfy either the descending or the ascending chain condition, they are nilpotent.

The present note deals mainly with rings of type $(\gamma)$. We show that they are all bound to $N$ and this yields the fact that every commutative ring is isomorphic to a subdirect sum of subdirectly irreducible rings some of which are fields, the others being bound to their maximal nilideals and therefore to their Jacobson radical. ${ }^{2}$

Presented to the Society, November 26, 1955; received by the editors November 5, 1955 and, in revised form, September 10, 1956.

1 Numbers in square brackets refer to the bibliography at the end of the paper.

2 This extends, in the commutative case, a result of Andrunakievic (Doklady Akademii Nauk. SSSR. (N.S.) vol. 98 (1954) pp. 329-332) that every ring with the descending chain condition on two-sided ideals, is a direct sum of a finite number of 
Furthermore we show that a ring of type $(\gamma)$ with either the descending or the ascending chain condition has $D=N$, thus extending a result of McCoy [1]; and that every such ring possesses a unity element. This gives us the fact that every commutative subdirectly irreducible ring with either the descending or the ascending chain condition is a field, is nilpotent or has a unity element and is a field modulo its maximal nilideal. Several other weaker conditions are shown to yield the existence of a unity element. Finally, a major part of the paper is devoted to an example of a ring of type $(\gamma)$ without a unity element.

1. The fundamental theorem on rings of type $(\gamma)$ is (see [1]): if:

TheOREM a. $A$ ring of type $(\gamma)$ is subdirectly irreducible if and only

1. The set of elements of $A$ that annihilate $D$ is precisely $Q, a$ principal ideal $=(j)$.

2. The set of elements of $A$ that annihilate $Q$ is precisely $D$.

3. The quotient ring $A-D$ is a field.

4. For every element $d$ in $D$, which is not in $Q$, there exists an element $d^{\prime}$ in $D, d^{\prime}$ not in $Q$, such that $d d^{\prime}=j$.

Using a technique of [2] we first prove:

TheOREM 1. Every subdirectly irreducible ring $A$ of type $(\gamma)$ is bound to its maximal nilideal $N$.

Proof. Let $x$ be any element in $N^{*}$. That is, $x N=0$. Then clearly, $x$ is in $D$. If $x$ is not in $N, x^{2}$ is not in $N$, and since $x$ is in $D, x^{2}$ is in $D$. By Theorem a, number 4 , there exists an element $x^{\prime \prime}$ in $D$ such that $x^{2} \cdot x^{\prime \prime}=j$. Consider the element $x \cdot x^{\prime \prime}$. It is in $N$ since $\left(x \cdot x^{\prime \prime}\right)^{2}=x^{2} \cdot x^{\prime \prime}$ $\cdot x^{\prime \prime}=j \cdot x^{\prime \prime}=0$, since $j$ annihilates $D$. However $x \cdot x x^{\prime \prime}=j \neq 0$ which contradicts $x N=0$. Therefore $x$ is in $N, N^{*} \leqq N, A$ is bound to $N$.

Since every subdirectly irreducible ring of type $(\beta)$ is bound to $N$, [2], we have

COROLLARY. Every subdirectly irreducible ring is either a field or is bound to its maximal nilideal.

We now turn our attention to the existence of a unity element and the relationship between $D$ and $N$.

simple rings with unit and a bound ring; as well as the result of Brown and McCoy (Proc. Amer. Math. Soc. vol. 1 (1950) pp. 165-171) that a ring with the descending chain condition on right ideals is a direct sum of a semisimple and a bound ring. This was done for finite dimensional algebras by Hall (Trans. Amer. Math. Soc. vol. 48 (1940) pp. 391-404). 
Lemma 1. For every subdirectly irreducible ring $A, A$ has a unity element if and only if $A-N$ has a nonzero idempotent.

Proof. One way is trivial. Conversely if $A-N$ has a nonzero idempotent then $A$ has a nonzero idempotent (see the more general result as part of Hall, Ann. of Math. vol. 40 (1939) p. 368, Theorem 6.1). Let $S$ be a nonzero ideal of $A$ which has this idempotent as a unity element. Then $A=S \oplus S^{*}$, where $S^{*}$ is the annihilator of $S$. Since $A$ is subdirectly irreducible and $S \neq 0, S^{*}=0$ and therefore $A=S, A$ has a unity element.

Corollary. If a subdirectly irreducible ring $A$ of type $(\gamma)$ has $D=N$ then $A$ has a unity element.

Proof. Since $A-D$ is a field (Theorem a, number 3 ), $A-D$ has a nonzero idempotent. Then if $N=D, A-N$ has one, and therefore by the lemma, $A$ has a unity element.

From [1] we learn that if $A$ is a subdirectly irreducible ring of type $(\gamma)$ and if $A$ has the descending chain condition, then $D=N$. By the above corollary, $A$ has a unity element. We now extend this to rings with the ascending chain condition.

TheOREM 2. If $A$ is a subdirectly irreducible ring of type ( $\gamma)$ and if $A$ has the ascending chain condition, then $D=N$.

Proof. If $D \neq N$, take $x$ in $D, x$ not in $N$. Then all the powers $x^{2}, x^{3}, \cdots, x^{n}, \cdots$ are in $D$ and not in $N$, and for every power $x^{n}$ there exists an element $y_{n}$ such that $x y_{1}=x^{2} y_{2}=\cdots=x^{n} y_{n}=\cdots=j$. Define

$$
V_{i}=\left\{z: z x^{i}=0\right\} .
$$

Clearly $V_{1}<V_{2}<V_{3}<\cdots<V_{n}<\cdots$. Also, since all the elements of $V_{i}$ are divisors of zero, $V_{i} \leqq D$, for every $i$. Since $x^{i} \cdot D \neq 0$ for every $i$ (since $x$ is not nilpotent), all the $V_{i}$ are properly contained in $D$. Now $y_{1}$ is not in $V_{1}$, but $x \cdot x y_{1}=x j=0$, and therefore $y_{1}$ is in $V_{2}$. Similarly $y_{n}$ is not in $V_{n}$, but $x \cdot x^{n} y_{n}=x j=0$, and therefore $y_{n}$ is in $V_{n+1}$. Therefore the $V_{i}$ form a properly ascending chain which contradicts the ascending chain condition. Therefore no such $x$ exists, and $D=N$. Therefore we have

Theorem 3. If $A$ is a subdirectly irreducible ring of type ( $\gamma)$ and if $A$ has either the descending or the ascending chain condition, then $A$ has $a$ unity element.

2. Examples. Since every subdirectly irreducible ring of type $(\beta)$ is bound to $N$ and is nilpotent if it has either the descending or the 
ascending chain condition we shall first give an example of such a ring which is not nilpotent. It is based on an example in [1].

Let $A$ be an algebra over a field of characteristic 2, with the following basis: $x, x^{2}, \cdots, x^{n}, \cdots ; x^{t-1}, x^{t-2}, \cdots, x^{t-m}, \cdots$, where both $x$ and $t$ are indeterminates and $x^{t}=0$. The algebra $A$ is the set of all finite linear combinations. Then the unique minimal ideal $Q=\left\{x^{t-1}\right\}$. Since $x^{t-1} \cdot\left(\sum \alpha_{i} \cdot x^{i}+\sum \beta_{j} \cdot x^{t-j}\right)=0$, every element is a divisor of zero. Thus $A$ is of type $(\beta)$. To see that $x^{t-1}$ is in fact contained in every ideal, let $z$ be any element of $A, z=\sum_{1}^{n} \alpha_{i} x^{i}+\sum_{1}^{m} \beta_{j} x^{t-j}$. If the $\alpha_{i}$ are not all zero, let $\alpha_{s}$ be the first nonzero $\alpha_{i}$ (and therefore $=1$ ) and consider $z \cdot x^{t-s-1}$. Since $x^{t-p} \cdot x^{t-q}=x^{t} \cdot x^{t-q-p}=0, z \cdot x^{t-s-1}=x^{s} \cdot x^{t-8-1}$ $+\alpha_{s+1} x^{s+1} \cdot x^{t-8-1}+\cdots+\alpha_{n} x^{n} x^{t-s-1}=x^{t-1}$. If on the other hand all the $\alpha_{i}$ are zero, $z=\sum_{1}^{m} \beta_{j} x^{t-j}$. Then if $\beta_{m}$ is the last nonzero coefficient (and therefore $=1$ ) consider $z \cdot x^{m-1}=\beta_{1} x^{t-1} x^{m-1}+\cdots+x^{t-m} x^{m-1}$ $=x^{t-1}$. Of course if $m=1, z=x^{t-1}$. Therefore $x^{t-1}$ is in every nonzero ideal, and $A$ is subdirectly irreducible. Finally we note that $x$ is not nilpotent and therefore $A$ is not nilpotent.

Turning back now to subdirectly irreducible rings of type $(\gamma)$ we give an example of such a ring without a unity element.

Let $A$ be an algebra over a field $F$, characteristic 0 , with the following basis: $e, e^{2}, \cdots, e^{n}, \cdots, z_{0}, z_{1}, z_{2}, \cdots, z_{m}, \cdots$, where $e$ is an indeterminate, and $A$ is the set of all finite linear combinations of the $e^{n}$ 's and $z_{m}$ 's with coefficients in $F$. The multiplication table is:

$$
\begin{aligned}
z_{i} \cdot z_{j} & =0, & \text { for every } i \text { and } j ; \\
e \cdot z_{0} & =z_{0} ; & \text { for } m>0 . \\
e \cdot z_{m} & =z_{m}+z_{m-1}, &
\end{aligned}
$$

We define $Q$ to be the ideal generated by $z_{0}$ and will show later that $Q$ is the unique minimal ideal contained in every nonzero ideal. The maximal nilideal $N$ is clearly the ideal generated by $z_{0}, z_{1}, \cdots$, $z_{m}, \cdots$, and $N^{2}=0$.

We define $D$ to be the set of all $\sum_{i=1}^{n} \alpha_{i} e^{i}+\sum_{j=0}^{m} \beta_{j} z_{j}$, where $\sum_{i=1}^{n} \alpha_{i}=0$. We will show later that $D$ is the set of all divisors of zero.

Clearly $A$ contains nonzero nilpotent elements. It also has nondivisors of zero, namely $e$. For a simple computation shows that if $e \cdot a=0$, where $a$ is any element in $A$, then $a$ must be zero. Consequently $A$ is of type $(\gamma)$.

To see that $A$ is subdirectly irreducible we shall first establish the four parts of Theorem a and then prove that $D$ is the set of all divisors of zero. 
(1) $\{x: x D=0\}=Q$.

Proof. Since $z_{0} \cdot z_{i}=0$ for every $i$, and since

$$
z_{0} \cdot \sum_{i=1}^{n} \alpha_{i} e^{i}=z_{0} \cdot \sum_{i=1}^{n} \alpha_{i}=0 \text { if } \sum_{i=1}^{n} \alpha_{i}=0,
$$

it is clear that $Q \leqq\{x: x D=0\}$. To show that $\{x: x D=0\} \leqq Q$, we must first establish the formula

$$
z_{m} \cdot\left(e^{2}-e\right)^{m}=z_{0}
$$

We show this by induction. For $m=1, z_{1} \cdot\left(e^{2}-e\right)^{1}=z_{1} \cdot e^{2}-z_{1} \cdot e$ $=z_{1}+2 z_{0}-z_{1}-z_{0}=z_{0}$. We assume that $z_{m-1} \cdot\left(e^{2}-e\right)^{m-1}=z_{0}$ and we consider $z_{m} \cdot\left(e^{2}-e\right)^{m}$. Since $z_{m} \cdot e=z_{m}+z_{m-1}, z_{m} \cdot(e-1)=z_{m-1}$, when we multiply by $e$ this becomes $z_{m} \cdot\left(e^{2}-e\right)=z_{m-1} \cdot e$. Therefore $z_{m} \cdot\left(e^{2}-e\right)^{m}$ $=z_{m} \cdot\left(e^{2}-e\right) \cdot\left(e^{2}-e\right)^{m-1}=z_{m-1} \cdot e \cdot\left(e^{2}-e\right)^{m-1}=e \cdot z_{0}=z_{0}$. This establishes (1). As a consequence of (1) we have

$$
z_{m} \cdot\left(e^{2}-e\right)^{n}=0,
$$

for $n>m$.

This is clear since $z_{m} \cdot\left(e^{2}-e\right)^{n}=z_{m} \cdot\left(e^{2}-e\right)^{m} \cdot\left(e^{2}-e\right)^{n-m}=z_{0} \cdot\left(e^{2}-e\right)^{n-m}$ $=0$, because $e^{2}-e$ is in $D$.

Now let $x$ be any element such that $x D=0 ; x=\sum_{1}^{n} \alpha_{i} e^{i}+\sum_{0}^{m} \beta_{j} z_{j}$. Since $\left(e^{2}-e\right)^{k}=e^{k} \cdot(e-1)^{k}$ has the sum of its coefficients $=1-{ }_{k} C_{1}$ $+{ }_{k} C_{2} \cdots \pm 1=(1-1)^{k}=0,\left(e^{2}-e\right)^{k}$ is in $D$ for every $k$. Take $k=m+1$ and consider $x \cdot\left(e^{2}-e\right)^{m+1}=0$. Since $\sum_{0}^{m} \beta_{j} z_{j} \cdot\left(e^{2}-e\right)^{m+1}=0$ by (2), we have $\sum_{1}^{n} \alpha_{i} e^{i} \cdot\left(e^{2}-e\right)^{m+1}=0$. This is possible only if all the $\alpha_{i}$ are zero, and therefore $x=\sum_{0}^{m} \beta_{j} z_{j}$. If there is a $\beta_{m} \neq 0$, with $m>0$, consider $0=x \cdot\left(e^{2}-e\right)^{m}=\sum_{0}^{m} \beta_{j} z_{j} \cdot\left(e^{2}-e\right)^{m}=\beta_{m} z_{0}$, by (2) and (1). Since this is impossible, all the $\beta_{m}$ 's, $m>0$, must be zero, and $x=\beta_{0} z_{0}, x$ is in $Q$.

(2) $\{x: x Q=0\}=D$.

Proof. As in the proof of (1) it is clear that $D \cdot Q=0$. To show that $\{x: x Q=0\} \leqq D$, let $x$ be any element such that $x Q=0, x=\sum_{1}^{n} \alpha_{i} e^{i}$ $+\sum_{0}^{m} \beta_{j} z_{j}$. Since $x Q=0, x z_{0}=0$ and therefore $\sum_{1}^{n} \alpha_{i} e^{i} \cdot z_{0}=z_{0} \cdot \sum_{1}^{n} \alpha_{i}$ $=0$. Thus $\sum_{1}^{n} \alpha_{i}=0$, and $x$ is in $D$.

(3) $A-D$ is a field.

Proof. Let $x$ be any element in $A, x=\sum_{1}^{n} \alpha_{i} e^{i}+\sum_{0}^{m} \beta_{j} z_{j}$. Then

$$
\begin{aligned}
x & =\sum_{1}^{n} \alpha_{i} \cdot e+\left(-\sum_{2}^{n} \alpha_{i} \cdot e+\sum_{2}^{n} \alpha_{i} \cdot e^{i}\right)+\sum_{0}^{m} \beta_{j} z_{j} \\
& =\alpha \cdot e+g(e)+\sum_{0}^{m} \beta_{j} z_{j},
\end{aligned}
$$

where $\alpha=\sum_{1}^{n} \alpha_{i}$ and where $g(e)+\sum_{0}^{m} \beta_{j} z_{j}$ is in $D$. Thus $x=\alpha e+d$, with $d$ in $D$. Therefore $A-D$ is simply the set of all $\alpha e+D, \alpha$ in $F$. 
Since $e-e^{2}$ is in $D, \alpha e \cdot e=\alpha \cdot e^{2} \equiv \alpha \cdot e$ (modulo $D$ ). Therefore $e+D$ is the unity element of $A-D$. Also $(\alpha e+D)\left(\alpha^{-1} \cdot e+D\right)=e^{2}+D=e+D$ and therefore $A-D$ is a field.

(4) If $d$ is in $D$, not in $Q$, there exists an element $d^{\prime}$ in $D$, not in $Q$, such that $d d^{\prime}=z_{0}$.

Proof. Let $x$ be any element in $D, x$ not in $Q$. Then $x=\sum_{1}^{n} \alpha_{i} e^{i}$ $+\sum_{0}^{m} \beta_{j} z_{j}$, with $\sum_{1}^{n} \alpha_{i}=0$. If all the $\alpha_{i}$ 's are zero, $x=\sum_{0}^{m} \beta_{j} z_{j}$, with $\beta_{m} \neq 0, m>0$. Let $x^{\prime}=\beta_{m}^{-1} \cdot\left(e^{2}-e\right)^{m}$. Then $x \cdot x^{\prime}=\sum_{0}^{m} \beta_{j} z_{j} \cdot \beta_{m}^{-1} \cdot\left(e^{2}-e\right)^{m}$ $=\beta_{m} z_{m} \beta_{m}^{-1} \cdot\left(e^{2}-e\right)^{m}$, by (2) and this is $=z_{0}$, by (1). Clearly $x^{\prime}$ is in $D$ and not in $Q$.

Therefore we assume that not all of the $\alpha_{i}$ 's are zero, $\alpha_{n} \neq 0$. Consider the following set of elements of $F$ :

$$
\begin{aligned}
& \gamma_{0}=\sum_{1}^{n} \alpha_{i}, \quad \gamma_{1}=\sum_{1}^{n}{ }_{i} C_{1} \cdot \alpha_{i}, \quad \gamma_{2}=\sum_{2}^{n}{ }_{i} C_{2} \cdot \alpha_{i}, \cdots \\
& \gamma_{8}=\sum_{s}^{n}{ }_{i} C_{s} \cdot \alpha_{i}, \cdots, \gamma_{n}=\sum_{n}^{n}{ }_{i} C_{n} \cdot \alpha_{i}=\alpha_{n} .
\end{aligned}
$$

Thus not all of the $\gamma_{\mathrm{s}}$ are zero and there exists an integer $r$ such that $\gamma_{0}=\gamma_{1}=\cdots=\gamma_{r-1}=0, \gamma_{r} \neq 0$. Let $x^{\prime}=\gamma_{r}^{-1} \cdot z_{r}$ and compute

$$
\begin{aligned}
\gamma_{r} \cdot x \cdot x^{\prime} & =x \cdot z_{r}=\sum_{1}^{n} \alpha_{i} e^{i} \cdot z_{r}+\sum_{0}^{m} \beta_{j} z_{j} \cdot z_{r}=\sum_{1}^{n} \alpha_{i} e^{i} \cdot z_{r} \\
& =\sum_{i=1}^{n} \sum_{j=0}^{r} \alpha_{i} \cdot{ }_{i} C_{j} \cdot z_{r-j}
\end{aligned}
$$

where ${ }_{i} C_{0}$ is taken to be 1 and where ${ }_{i} C_{j}$, with $i<j$, is taken to be 0 . Then

$$
\gamma_{r} \cdot x \cdot x^{\prime}=z_{r} \cdot \gamma_{0}+z_{r-1} \cdot \gamma_{1}+\cdots+z_{1} \gamma_{r-1}+z_{0} \gamma_{r}=z_{0} \gamma_{r} .
$$

Therefore $x \cdot x^{\prime}=z_{0}$. This establishes (4).

To see that $D$ is the set of all divisors of zero it is sufficient to show that every divisor of zero is in $D$, since we already know that $D \cdot z_{0}=0$. Thus let $x$ be any divisor of zero. Then for some $y, x y=0$. If $x$ is not in $D$, then $y$ must be in $D$, because $A-D$ is a field. If $y$ is in $Q$, $y=\alpha z_{0}$, then $x \cdot z_{0}=0$ and $x Q=0$. By (2), $x$ is in $D$. If $y$ is not in $Q$, then by (4), there exists an element $y^{\prime}$ in $D$ such that $y y^{\prime}=z_{0}$. Then $x y y^{\prime}=x z_{0}=0, x Q=0, x$ is in $D$ by (2).

Therefore by Theorem a, $A$ is a subdirectly irreducible ring.

We must finally prove that $A$ does not possess a unity element. If $f$ is a unity element then it must be congruent to $e$ modulo $D, f=e+d$. Since $e$ is not a unity element $\left(e \cdot z_{1}=z_{1}+z_{0} \neq z_{1}\right), d \neq 0$. Then there 
exists an element $d^{\prime}$ such that $d d^{\prime}=z_{0}$ and therefore $f d^{\prime}=e d^{\prime}+z_{0}=d^{\prime}$. That is, $e d^{\prime}-d^{\prime}=-z_{0}$. Now $d=\sum_{1}^{n} \alpha_{i} e^{i}+\sum_{0}^{m} \beta_{j} z_{j}$, with $\sum_{1}^{n} \alpha_{i}=0$. If all the $\alpha_{i}$ are zero, then $d^{\prime}$ can be taken $=-\beta_{m}^{-1} \cdot\left(e^{2}-e\right)^{m}$, (see proof of (4)). Consequently $e \cdot \beta_{m}^{-1} \cdot\left(e^{2}-e\right)^{m}-\beta_{m}^{-1} \cdot\left(e^{2}-e\right)^{m}=-z_{0}, \quad\left(e^{2}-e\right)^{m}$ $\cdot(e-1)=-\beta_{m} z_{0}$. Multiplying by $e \cdot z_{m+1}$ this becomes $\left(e^{2}-e\right)^{m+1} \cdot z_{m+1}$ $=z_{0}=-\beta_{m} z_{0} z_{m+1} \cdot e=0$, which is impossible.

On the other hand if the $\alpha_{i}$ are not all zero then $d^{\prime}$ can be taken $=\gamma_{r}^{-1} \cdot z_{r}$, (see proof of (4)), and we have $e \cdot \gamma_{r}^{-1} \cdot z_{r}-\gamma_{r}^{-1} \cdot z_{r}=-z_{0}$. Therefore $z_{r}-e z_{r}=\gamma_{r} z_{0}=z_{r}-z_{r}-z_{r-1}=-z_{r-1}$. This is false unless $r=1$, $d^{\prime}=z_{1}, f=e+e-e^{2}+\sum_{0}^{m} \beta_{j} z_{j}$. Then $f \cdot z_{2}=2 e \cdot z_{2}-e^{2} z_{2}=2 z_{2}+2 z_{1}-z_{2}$ $-2 z_{1}-z_{0}=z_{2}-z_{0} \neq z_{2}$. Therefore $A$ has no unity element.

We might point out that for subdirectly irreducible rings of type $(\gamma), D$ is a maximal regular ideal and therefore contains $J$, the Jacobson radical. In this particular example, $J=N$, since $A-N$ is the set of all finite sums $\sum_{1}^{n} \alpha_{i} e^{i}$ and this set has zero Jacobson radical.

Since rings of type $(\beta)$ and $(\gamma)$ seem to have such similar properties, one might expect a more intimate relationship between them. However the $N$ of a ring of type $(\gamma)$ is not necessarily subdirectly irreducible, as the main example above proves.

One can however show that every ring of type $(\beta)$ is contained in a ring of type $(\gamma)$. The following theorem was pointed out to the author by Professor McCoy. It can be proved for the noncommutative case, and shows further that a ring of type $(\gamma)$ without a unity element is contained in a ring of type $(\gamma)$ with a unity element.

THEOREM 4. Every subdirectly irreducible ring without a unity element, can be embedded in a subdirectly irreducible ring with a unity element.

As Professor McCoy pointed out, the proof of this theorem follows almost immediately from Lemma 5 in the paper, On the theory of radicals in a ring, by M. Nagata, Journal of the Mathematical Society of Japan vol. 3 (1951) pp. 330-344.

\section{BiBLIOGRAPHY}

1. Neil McCoy, Subdirectly irreducible commutative rings, Duke Math. J. vol. 12 (1945) pp. 381-387.

2. Nathan Divinsky, Pseudo-regularity, Canadian Journal of Mathematics vol. 7 (1955) pp. 401-410.

\section{UNIVERSITY OF MANITOBA AND}

Research Institute of the Canadian Mathematical Congress 